Meta

Journal des tradlucteurs

Translators' Journal

\title{
Temps forts ou temps faibles pour la terminologie en entreprise?
}

\section{Nada Kerpan}

Volume 36, numéro 1, mars 1991

La terminologie dans le monde : orientations et recherches

URI : https://id.erudit.org/iderudit/003303ar

Aller au sommaire du numéro

Éditeur(s)

Les Presses de l'Université de Montréal

ISSN

0026-0452 (imprimé)

Découvrir la revue

Citer cet article

Kerpan, N. (1991). Temps forts ou temps faibles pour la terminologie en entreprise? Meta, 36(1), 234-239. d'utilisation que vous pouvez consulter en ligne.

https://apropos.erudit.org/fr/usagers/politique-dutilisation/ 


\section{TEMPS FORTS OU TEMPS FAIBLES POUR LA TERMINOLOGIE EN ENTREPRISE?}

NADA KERPAN

Bell Canada, Montréal, Canada

L'action de traducteurs avertis, de linguistes innovateurs et de terminologues actifs a permis d'établir, au cours des vingt-cinq dernières années, la fonction et la place de la terminologie dans la communication spécialisée. Par ailleurs, la formation à cette discipline et aux langues de spécialité, assurée dans le cadre des études de traduction ou de linguistique, a familiarisé les esprits avec la réalité et la nécessité terminologiques.

La terminologie n'est plus, dans l'entreprise d'aujourd'hui, une inconnue. Elle ne fait pas, non plus, figure d'intrus ou de gêneur, perturbant, comme on était prompt à le penser jadis, l'univers de la traduction et celui du traducteur.

Est-ce à dire que, dans l'entreprise de 1990, la terminologie occupe bien la place qui est la sienne, qu'elle fonctionne à plein régime, sans heurts et sans à-coups? Ou connaît-elle de la turbulence et une paupérisation, qui en fera une profession de servitude, aléatoire, disloquée? Ou enfin la situation se résume-t-elle à un laborieux équilibre entre le consensus et le dissensus, entre la demande et les moyens?

\section{LA DEMANDE DE TERMINOLOGIE}

Dans le secteur privé ou parapublic, la demande théorique de services linguistiques - traduction, rédaction et terminologie - est énorme et demeure fonction directe de la francisation. La demande réelle, fortement tributaire de la dynamique même de l'entreprise, a atteint des degrés impressionnants. Dans la moyenne et la grande entreprise, les besoins en services linguistiques ont, en effet, connu une croissance considérable et soutenue. Ainsi, en seule traduction, la prestation peut, dans certaines sociétés, se chiffrer jusqu'à dix millions de mots par année, masse abondamment composée de textes à caractère spécialisé, soit technique, scientifique, commercial ou administratif. C'est donc dire que la traduction - la forme la plus opérante, jusqu'ici, de la francisation au Canada et particulièrement au Québec - constitue le canal et le demandeur le plus important et le plus régulier de terminologie.

Les besoins en terminologie sont étroitement liés aux besoins en traduction. Mais ils se manifestent aussi par une demande directe, de la part des personnels d'entreprise, de terminologie thématique ou ponctuelle destinée à servir diverses formes de communication écrite et orale - rédaction spécialisée, formation, par exemple.

De plus, ces besoins sont multiples. Non seulement ils concernent la ou les spécialités de l'entreprise, mais encore tous les aspects de son fonctionnement général et courant - gestion du personnel, gestion financière, etc.

Comment expliquer que la demande persiste de façon aussi marquée alors que la francisation devrait être largement réalisée? 


\section{LE TRONC COMMUN}

Le tronc commun de terminologies n'est-il pas aujourd'hui acquis, bien intégré et adéquat?

$\mathrm{Au}$ cours des années, les volontés et les énergies ralliées tant dans le secteur public que privé n'ont pas produit un tronc commun complet, uniforme et définitif. Certes, le volume de terminologies communes disponibles est imposant; malgré ce qu'on peut prétendre, ces terminologies ne sont pas exhaustives, ni nécessairement fixées ou normalisées. Si elles comportent une part stable, elles sont sujettes au changement, à l'évolution et à l'expansion - à preuve, le domaine des relations du travail ou celui de la comptabilité. La dynamique normale de la langue et les personnels qui les utilisent les modifient sur le plan tout autant langagier que technique. En effet, à une tendance pour le nouveau et le renouveau s'ajoutent des causes tels un manque de formation ou de rigueur chez les usagers techniques et même langagiers ou encore une absence de souci normalisateur.

De plus, en de nombreuses circonstances, il faut tout simplement déplorer des lacunes dans la diffusion ou un accès difficile à ces vocabulaires communs.

Par ailleurs, des domaines, auparavant fermés ou hautement spécialisés, se sont ouverts à divers publics, voire au grand public et connaissent une effervescence tenace. L'informatique et les télécommunications demeurent, à cet égard, des exemples patents.

Le tronc commun de terminologies n'échappe donc pas à une demande d'intervention terminologique. Il réclame, encore aujourd'hui, une recherche plus poussée dans les divers domaines, la production de terminologies comparées, le traitement de la néologie, une harmonisation ou normalisation à petite échelle ou à l'échelle internationale.

\section{LES SPÉCIALITÉS CONNUES ET NOUVELLES}

Si spécifiques et maîtrisées soient-elles, les spécialités des entreprises sont des foyers d'évolution continue, pouvant se subdiviser et se parcelliser presque à l'envi; de nouveaux secteurs et sous-secteurs se créent. En effet, l'objectif d'innovation, la modernisation des techniques et la dynamique commerciale ne cessent de provoquer des changements. Même les domaines traditionnellement considérés comme bien établis ou, en apparence, moins sensibles aux transformations ne sont pas à l'abri de mutations. La manutention, le transport ferroviaire, l'exploitation forestière, la comptabilité, la téléphonie, l'informatique, la bureautique illustrent, à des degrés divers, l'inéluctabilité du changement.

Les spécialités constituent donc des zones actives de terminologie. Transmise le plus souvent par la documentation anglaise, la demande terminologique vise d'abord l'établissement de termes français, moins à l'étape de recherche-développement qu'à celles de production et de logistique. Mais apparât aussi le besoin de rationaliser, par l'uniformisation ou la normalisation, ces microcosmes terminologiques unilingues ou bilingues, complexes par la technicité ou la spécialisation, bouillonnants de néologie, ou encore surabondants par la synonymie.

De leur côté, les technologies nouvelles - pour ne citer que les biotechnologies sont de généreux producteurs et de forts demandeurs de terminologie. Milieux essentiellement créateurs et évolutifs, elles favorisent, de toute évidence, une éclosion souvent anarchique - de terminologies. Tout comme il n'y a pas de temps d'arrêt pour le progrès technique, de même les domaines de pointe n'offrent pas de répit à la langue, chargée d'en nommer les éléments.

Qu'il s'agisse de spécialités connues ou nouvelles, la demande est constante et pressante. 


\section{L'OFFRE DE TERMINOLOGIE}

À la demande correspond bel et bien une offre terminologique. Importante, cette offre s'avère multiple par la provenance, complémentaire, répétitive et, à l'occasion, divergente. Elle est constituée des travaux menés, depuis de nombreuses années, par les centres de recherche publics, par les entreprises et organismes divers, de même que par les particuliers. C'est sous forme de publications et par voie électronique pour ce qui est notamment des banques publiques (celles de l'Office de la langue française et du Secrétariat d'État), qu'elle est diffusée; mais elle reste, en plusieurs cas, confinée au milieu immédiat de recherche terminologique.

On constate, dans les faits, une certaine fluidité de production terminologique tout autant dans des domaines jugés classiques telles la fiscalité, la comptabilité, par exemple, que dans des secteurs nouveaux telles les biotechnologies, l'intelligence artificielle, la bureautique, la télématique, la robotique.

Cependant, l'offre réelle n'est pas en adéquation véritable avec les besoins. Elle est soit insuffisante, inexistante au moment voulu, de qualité inégale ou carrément douteuse, ou pouvant même être pléthorique, laissant le demandeur aux prises avec de nombreuses solutions.

\section{LE TRAITEMENT DE LA DEMANDE}

Comment la terminologie s'exerce-t-elle, en 1990, en milieu d'entreprise?

\section{LE TERMINOLOGUE ET LA QUANTITÉ}

Plusieurs entreprises, notamment les très grandes, disposent d'un service pouvant compter jusqu'à dix terminologues ou réalisant une part de leur demande terminologique par la formule du contrat. Ressources importantes, dira-t-on, mais elles sont en général, affectées à des fonctions variées, dont, entre autres, le soutien au personnel et aux traducteurs par la terminologie ponctuelle, la recherche thématisée ou thématique, et le conseil linguistique.

Certaines de ces grandes sociétés concentrent leurs efforts sur des projets de terminologie systématique, onéreux en temps mais déjà rentables à court terme, quoi qu'en pensent certains gestionnaires. Elles ne consacrent à la ponctuelle ou au conseil linguistique qu'une faible part de leur personnel, soit parce que la demande ne le justifie pas, soit parce que la recherche systématique est l'orientation privilégiée. D'autres continuent d'être des producteurs diversifiés tout autant de ponctuelle, de thématisée que de thématique.

Dans ces milieux, en apparence bien pourvus en effectif terminologique, les besoins ne cessent de dépasser les moyens - cas des secteurs de l'informatique et des télécommunications, par exemple. C'est, en effet, par bribes, par pièces, par ensembles réduits que s'effectue, à un rythme non adapté à la demande, la terminologie de l'activité fondamentale de l'entreprise et de son exploitation courante.

La moyenne entreprise, pour sa part, ne compte pas nécessairement de terminologues. La petite entreprise, elle, ne peut s'offrir de tels spécialistes.

\section{LE TERMINOLOGUE ET LA QUALITÉ}

La qualité ne semble pas susciter d'insatisfaction ou de blâme véritable, sinon celui de paraître à certains comme étant du travail long, universitaire ou intellectuel. En dépit des contraintes de temps, de la complexité des domaines, de l'ampleur et de la ténacité de la demande, la terminologie thématique produite par des terminologues confirmés se révèle de bonne ou d'excellence qualité. Cette réussite s'explique par le respect et la maîtrise du processus reconnu de recherche, par une association étroite avec les 
personnels spécialisés, par le travail en comité intraentreprise ou interentreprise ainsi que par un objectif d'uniformisation ou de normalisation.

De son côté, la recherche ponctuelle ou thématisée fournit une terminologie plus rapide et relativement satisfaisante puisque, de façon générale, elle est accomplie par des terminologues consciencieux. Toutefois, cette terminologie en garde les inconvénients propres : réalisée à la pièce, avec une certaine précipitation, et sans perspective globale ou pleinement réfléchie sur le sujet. Aussi, si un tel produit est utile aux demandeurs ponctuels que sont le traducteur ou le personnel d'entreprise, il n'offre pas la même certitude ou exhaustivité que les termes obtenus par la voie systématique. Il faut préciser, par ailleurs, que nombreux sont les termes qui s'implantent par ce procédé et ce, non seulement en ce qui touche l'activité propre de l'entreprise, mais aussi les autres secteurs de son exploitation courante. Il faut également rappeler qu'un lexique ou un vocabulaire établi à partir de travaux ponctuels ne présente pas la même qualité d'information ni la même crédibilité que le résultat d'une recherche thématique.

\section{LE TRADUCTEUR ET LA QUANTITÉ}

Le plus gros de la demande terminologique est traité par le traducteur. La situation s'explique par l'absence de terminologues, par une délégation totale de la fonction terminologie au secteur de la traduction, par une autonomie ou une volonté d'autonomie chez le traducteur, ou encore par un trop-plein de la demande même lorsque le traducteur dispose d'un soutien terminologique. Quoi qu'il en soit, le caractère professionnel de la traduction ne commande-t-il pas une intervention et une production terminologiques chez le traducteur? D'ailleurs, l'enseignement actuel de la traduction fait une part à la terminologie.

Le temps consacré par chaque traducteur aux recherches terminologiques serait de l'ordre de $45 \%$ de ses énergies 1 .

En effet, la variété et la nouveauté des domaines abordés, la quantité et la complexité des travaux plongent le traducteur dans la réalité concrète de la terminologie. Le pourcentage avancé ne saurait, toutefois, se confirmer que dans les milieux authentiquement professionnels, sensibilisés aux langues de spécialité. Il va de soi qu'une telle proportion ne correspond pas exclusivement à une production terminologique, mais qu'elle comprend, en bonne partie, la simple consultation d'ouvrages terminologiques. Enfin, la quantité de termes que les traducteurs mettent au point dans le cadre de leurs textes ainsi que le nombre de lexiques et vocabulaires qu'ils dressent par le dépouillement de leurs travaux témoignent de leurs activités terminologiques.

\section{LE TRADUCTEUR ET LA QUALITÉ}

Si la quantité de terminologie traitée par le traducteur est importante, la qualité se situe, pour sa part, à des niveaux divers: elle va de l'excellence à la médiocrité.

Il est des traducteurs maitres des fonctions que sont la traduction spécialisée et la terminologie proprement dite. Avant de procéder à l'opération effective de traduction, ils établissent, par un processus sérieux de documentation, la langue spécialisée de leurs textes. Ainsi dégagent-ils une terminologie efficace qui, par le pouvoir multiplicateur de la diffusion, prend racine et occupe le terrain dans le ou les domaines abordés. La qualité de la traduction et celle de la communication spécialisée en dépendent étroitement.

Cependant, il est des circonstances qui, dans le quotidien, s'accommodent mal d'une telle étape. En effet, sollicité par une demande ininterrompue et complexe et ne disposant pas du temps nécessaire, le traducteur doit, en maintes occasions, agir hâtivement face à des terminologies nouvelles, lacunaires ou non disponibles. La qualité de la terminologie ne peut que s'en ressentir. 
En outre, malgré la place officiellement faite à la terminologie depuis vingt ans, il existe encore des traducteurs qui n'en ont cure. Ou ils refusent de s'initier à fond au processus, ou ils refusent de le pratiquer faute de temps, de moyens ou d'intérêt. Les textes intègrent des terminologies approximatives, subjectives ou carrément de traduction, et souvent parallèles à des vocabulaires existants ou en voie d'établissement. L'accumulation de tels cas perturbe la communication spécialisée, dissocie les fonctions étroitement reliées que sont la traduction spécialisée et les langues de spécialité, et «déprofessionnalise» la traduction même.

\section{LE SPÉCIALISTE ET LE PERSONNEL D'ENTREPRISE}

Les divers personnels de l'entreprise sont, quant à eux et selon le cas, soit consommateurs soit producteurs de terminologie commune ou spécialisée. Les législations linguistiques, de même que les langagiers œuvrant notamment au sein de comités intraentreprises et interentreprises, les ont, dans une certaine mesure, sensibilisés au fait terminologique. De son côté, la mondialisation de la communication spécialisée les a éveillés au besoin d'une certaine uniformisation et cohérence en la matière.

Toutefois, la nouveauté ou l'indisponibilité de certaines terminologies et l'initiative personnelle amènent souvent ces groupes à modifier et à rejeter les termes fournis par les langagiers et à créer ainsi leur propre version terminologique, qu'il s'agisse de leur spécialité ou de domaines du tronc commun. Le procédé employé tient, en général, du calque ou de la dénomination subjective.

Le refus de terminologies communiquées par les langagiers s'exprime par diverses réactions: termes inusités risquant de gêner la communication, termes à coloration pédante, savante ou recherchée, ou termes déjà implantés même s'ils sont blâmables. La documentation anglaise constitue en général, on s'en doute bien, la trame de l'esprit et des habitudes du spécialiste et du personnel d'entreprise.

Dans l'entreprise, les spécialistes et les divers personnels sont de forts intervenants en matière de terminologies communes ou sectorielles; ils se posent en puissants décideurs et contrôleurs de terminologie. Bien associés aux langagiers, ils demeurent les meilleurs diffuseurs de terminologie et les agents les plus naturels de la communication spécialisée. Aussi les langagiers doivent-ils avoir partie liée avec eux et être d'une belle réceptivité, ténacité et même pugnacité.

\section{LE GESTIONNAIRE}

La terminologie n'a pas réussi à convaincre de sa légitimité tous les gestionnaires de services linguistiques. Pour certains, elle demeure une considération inutile et un service superflu. À leur avis, le traducteur s'en occupera si besoin est. Elle ne saurait être une activité distincte, comportant ses propres règles et contraintes, ni encore moins le fait de professionnels nommés terminologues.

Les gestionnaires à formation langagière ne sont pas nécessairement tous acquis à l'optique terminologique. Certains estiment qu'il s'agit là d'une activité intellectuelle ou d'un espace de pouvoir conquis par les terminologues et enlevé aux traducteurs. D'autres ont l'intelligence de la situation et n'hésitent pas à dégager les moyens pour répondre à une demande non équivoque et dont ils évaluent le bien-fondé même à court terme.

De leur côté, les gestionnaires sans spécialisation langagière ne sont pas d'office opposés à l'activité terminologique. Si d'aucuns s'étonnent du processus, ils savent en reconnaître la nécessité. D'autres, cependant, agissent comme des éléments démobilisateurs et déstabilisateurs en traduction professionnelle. 
En tout état de cause, lorsqu'il faut opérer une réduction de personnel ou de moyens, c'est quasi infailliblement le secteur de la terminologie - personnel ou fonction - qui en subit, le premier, les effets.

\section{PERSPECTIVE D'ENSEMBLE}

Ce ne sont pas les contraintes administratives, les ignorances ou les préjugés qui auront raison de la terminologie. La terminologie est une réalité de terrain, la matière vive de toute activité spécialisée; elle reste donc au cour de la traduction et de la rédaction spécialisées. Mais, même lorsqu'elle fait des avancées (dont, par exemple, son informatisation dans l'entreprise), elle n'est pas à l'abri de pressions diverses. Aussi les langagiers doivent-ils bien en comprendre les vertus pour mieux la pratiquer et mieux la réaffirmer, particulièrement auprès de ses détracteurs. Tôt ou tard, surtout à l'ère de la mondialisation des marchés, un recentrage s'imposera; la communication spécialisée ne peut supporter d'être brouillée par une optique non terminologique. Les mouvements d'uniformisation intraentreprise ou interentreprise, de normalisation nationale ou internationale en sont de justes témoignages.

NOTE

1. Groupe de travail interinstitutionnel «Terminologie - Documentation» (GITT)(1988): «La terminologie, pour quoi faire?», Terminologie et Traduction, 1, Commission des Communautés européennes, Bruxelles, pp. 5-9. 